\title{
Disablement Model Case Study: Running with Postural Orthostatic Tachycardia Syndrome
}

Jordan Fenney MS, LAT, ATC, CES* $\dagger$; Wendy Reitz LAT, ATC*; Hayley M. Ericksen PhD, LAT, ATC**

*Aurora Sports Health, West Bend, WI; **University of Wisconsin-Milwaukee, Milwaukee, WI; †Muscle and Movement Therapy, Cedarburg, WI

\section{ABSTRACT}

A 17-year-old high school female cross-country runner and basketball player presented with syncope following longendurance exercise. The syncope episodes started when the patient was 13 years old during a basketball game. After the first episode, the patient fainted every time she crossed the finish line of a cross-country meet. Her symptoms included increased heart rate, shortness of breath, and paresthesia in her hands and legs during exercise. The patient also experienced some dizziness when quickly sitting or standing during activities of daily living. The patient was first misdiagnosed with exercise induced asthma and prescribed a rescue inhaler to take prior to competition races, however the syncope episodes persisted. A referral was made to a cardiologist who performed the $Q$ sweat response (QSR) and tilt table tests. The test results, and clinical symptoms were consistent with a diagnosis of Postural Orthostatic Tachycardia Syndrome (POTS). Metoprolol was prescribed to slow her heart rate and fludrocortisone to increase blood volume. The patient took these medications daily and also took an extra half-a-tablet of metoprolol before exercising in hot conditions. The patient's syncope following long endurance races was managed by a coach who would catch the patient after she crossed the finish line, lay her down and elevate her legs until she regained consciousness. Once consciousness was regained, her heart rate and oxygen were monitored using a pulse oximeter. POTS is a unique condition that can be managed with strong communication between healthcare professionals. Proper management allows for continued competition with some modifications made by the athlete and close monitoring by the athletic trainer. It is important for athletic trainers to be educated on the signs and symptoms of POTS and understand that it can affect each athlete differently.

Key Phrases

Diagnostic testing and physical examination: nonmusculoskeletal conditions; interprofessional practice; secondary schools patient population

\section{Correspondence}

Dr. Hayley Ericksen, 3409 N. Downer Ave, Milwaukee, WI 53201.

E-mail: erickseh@uwm.edu

Twitter: @hayericksen_atc

\section{Full Citation}

Feeney J, Reitz W, Ericksen HM. Disablement model case study: running with postural orthostatic tachycardia syndrome. Clin Pract Athl Train. $2021 ; 4(2)$ :

https://doi.org/10.31622/2021/0004.2.7.

Submitted: October 29, 2020 Accepted: February 9, 2021.

Copyright (C) by Indiana State University

All rights reserved. ISSN Online 2577-8188

\section{INTRODUCTION}

$P$ ostural Orthostatic Tachycardia Syndrome (POTS) is an autonomic nervous system (ANS) disorder which can cause symptoms the following symptoms: light-headedness, fatigue, sweating, anxiety, heart palpitations, exercise intolerance and near syncope when standing. ' POTS is a unique condition affecting approximately 500,000 people in the United States, with predominance in young females. ${ }^{2}$ There are two classifications associated with POTS: primary POTS and secondary POTS. Primary POTS is thought to be idiopathic, occurring on its own without association with another disease, whereas with secondary POTS symptoms are experienced as a results of another disease diagnosis. ${ }^{3}$ Primary POTS can be classified deeper into partial dysautonomia and hyperadrengic. ${ }^{3}$ Proper classification of POTS can help with better management of the patient's symptoms. Clinicians should conduct a thorough history to determine if a patient may be experiencing primary or secondary POTS.

When the ANS is functioning properly, orthostatic stability is maintained when moving from supine or prone to seated by increasing heart rate by 10 to 20 beats per minute (bpm). ${ }^{1}$ The increase in heart rate allows the body to maintain blood pressure and supply oxygenated blood to the brain and other vital organs. ${ }^{1}$ This orthostatic stability is achieved within 60 seconds under normal conditions.

In a patient diagnosed with POTS, the innervation of the veins or the vein's response to sympathetic stimulation is impaired. ${ }^{1}$ This dysfunction leads to 
over-dilation of the blood vessels and venous pooling in the legs, thereby reducing venous return to the heart, which subsequently reduces the arterial flow of oxygenated blood to the brain.1,4 For approximately 10 minutes after standing, someone with POTS will experience a heart rate increase of $30 \mathrm{bpm}$ or more resulting in a heart rate greater than $120 \mathrm{bpm}$ as the ANS attempts to increase cardiac output and blood pressure to supply the brain and vital organs with oxygenated blood. With the increase in heart rate, a decrease in blood pressure can occur. This will lead to less blood flow to the brain and result in symptoms such as light-headedness, fatigue, sweating, anxiety, palpitations, exercise intolerance, and in some cases syncope. ${ }^{1}$ Symptoms of POTS can impair a patient's ability to engage in physical activity and activities of daily living, and therefore, can greatly affect the patient's overall quality of life.

\section{PATIENT INFORMATION}

A 17-year-old female cross-country runner and basketball player presented with syncope following long-endurance exercise. The patient's other symptoms included increased heart rate, shortness of breath, and paresthesia in her hands and legs during and after exercise. The syncope episodes first started during a middle school basketball game in 2014, at the age of 13 years old. The patient stated that while playing in the game, she was running backwards and she tripped and fell. When she got back up, she had trouble breathing, so she was substituted out of the game. Her mother came down from the bleachers and as they were walking out of the gym, the patient fainted. The patient's mother took her to a primary care physician where the patient was first misdiagnosed with exercise induced asthma and given a rescue inhaler. The following year, at the age of 14, the patient used the prescribed rescue inhaler following cross-country practices when she continued to experience symptoms of increased heart rate, shortness of

Copyright $(\subset)$ by Indiana State University All rights reserved. ISSN Online 2577-8188 breath, and paresthesia in her hands and legs. The inhaler treatment provided no symptom relief. The patient returned to her primary care physician and was referred to a specialist at a children's hospital where she was then misdiagnosed with a "once in a lifetime throat spasm." The patient continued to participate in cross-country, but when she had two more syncope episodes during practice, the coach and athletic trainer decided that she would be unable to continue participation without a proper diagnosis. The patient was finally referred to a cardiologist who specializes in POTS and the correct diagnosis was made in 2016 , two years after her symptoms began.

\section{Activity and Participation}

Throughout the patient's high school career, she competed in two sports: basketball and cross country. The symptoms of her POTS diagnosis impacted her participation in practices and competition in different ways. Throughout her freshman and sophomore year, she had fainting spells during all activity until she received a proper diagnosis in 2016. With the correct diagnosis and medication, most of her symptoms were under control; however, one symptom, syncope, persisted and continued to plague her at the end of each cross-country competition. She was able to finish her race but would faint into the arms of her coach at the finish line. The nature of a cross-country finish line- an abrupt stop, caused a quick drop in the patient's blood pressure, which decreased blood flow to the brain and triggered a syncope episode. After which, the patient explained that she felt numbness and tingling in her hands and feet for about ten minutes before her symptoms would improve. During basketball practices and games, the patient did not experience syncope episodes but did have increased heart rate, shortness of breath, and paresthesia in hands and feet. When she began having these symptoms, she was able to stop participating, sit down, and hydrate while slowly decreasing her heart rate, thus avoiding a 
syncope episode. The patient was able to work through and manage her symptoms with proper recognition and recovery techniques. The support of medications and other modifications to her routine also helped her to manage her symptoms and allowed her to continue participating in physical activity.

\section{Differential Diagnosis and Evaluation}

The differential diagnosis list for this case included: tachycardia syndrome, chronic fatigue syndrome, anxiety, asthma, vasovagal syncope, orthostatic hypotension, or cardiac arrhythmias. The patient was first misdiagnosed with exercise induced asthma and treated with a rescue inhaler which did not improve her symptoms. The patient then saw a cardiologist, whom after evaluating her signs and symptoms, performed a series of tests to help determine a correct diagnosis. The cardiologist was able to rule out chronic fatigue syndrome, anxiety and orthostatic hypotension based on evaluation of the patient's signs and symptoms. The patient's EKG results were unremarkable, which helped rule out tachycardia syndrome and cardiac arrhythmias. The cardiologist suspected she may be suffering from POTS and ordered several tests to confirm the diagnosis. The $Q$ sweat response (QSR) test was performed to evaluate the sweat response of the sympathetic nervous system. The QSR test uses iontophoresis to stimulate the sweat glands to release acetylcholine, resulting in and increased sweat response. ${ }^{5}$ The standard testing sites include forearm, proximal leg, distal legs, and dorsum of the foot. ${ }^{6}$ The amount of sweat is measured by the change in humidity in the sweat capsule from baseline to 15 minutes post stimulation. ${ }^{6}$ In normal individuals, the sweat output increases for about 5 minutes until it reaches an inflection point and then slowly decreases. If a patient has a loss of sympathetic nerve terminals, the terminals will not release as much acetylcholine resulting in decreased sweat. ${ }^{5}$ The results of this patient's QSR test showed a normal volume of sweat on the

Copyright $(\subsetneq$ by Indiana State University All rights reserved. ISSN Online 2577-8188 forearm and proximal leg, increased volume on the distal leg, and decreased volume of sweat on the foot compared to the proximal leg. The decreased volume of sweat on the foot compared to the proximal leg shows that the patient has a sympathetic nervous system abnormality. The cardiologist summarized these results to indicate abnormal postganglionic sudomotor function showing a mild impairment in the autonomic nerves, a result that is consistent with a diagnosis of POTS.

The tilt table test measures heart rate and blood pressure while the body is in different positions.7 The test is meant to mimic the sudden change in posture resulting in syncope. The test begins with a 10-15 minute baseline period of lying in a supine position.7 The table is slowly raised to seventy degrees while blood pressure and heart rate are measured. 7 With the tilt table test the doctor is looking for two changes, a decrease in blood pressure and/or heart rate as the table is tilted upright. ${ }^{7}$ If the patient can lay at seventy degrees without symptoms the clinician will introduce a sympathetic medication called isoproterenol. This patient was tested with a sympathetic medication that caused the heart to beat stronger and faster. The patient's results with the medication showed an abnormal response to seventy degrees head-up tilt, increased heart rate during the second ten minutes of upright tilt, and variable blood pressure during upright tilt compared to supine baseline. Her tilt table test showed that she has hyperadrenergic POTS because her results varied after the sympathetic drug was introduced. The cardiologist's summary of these findings stated that the patient had normal cardiovagal function, but she was abnormal when the medication was introduced. This is consistent with the fact that she only experienced syncope episodes when under stress such as long-endurance events.

Body Structure and Function 
POTS affects the ANS, which is the reflexive and involuntary division of the central nervous system. ${ }^{8}$ It is responsible for conducting nerve impulses from the central nervous system to cardiac muscle, smooth muscle, and glands. ${ }^{8}$ The basic functions of the ANS include regulation of one's heart rate and contraction of smooth muscle in the digestive tract. 8 POTS causes an exaggerated sympathetic response when changing orthostatic positioning.

\section{Environmental and Personal Factors}

Additional factors that may exacerbate the symptoms of POTS include, decreased fluid intake, dehydration, exercise, morning hours, fever and high ambient temperatures. ${ }^{7}$ Prior to running in hot conditions, the patient would take an extra dose of Metoprolol. In extreme heat, the sympathetic nervous system dilates the blood vessels, which brings the blood closer to the skin's surface and heat is lost through radiation from the body's surface. The body also reacts to heat by sending a signal, via the sympathetic nerves, to the sweat glands in the skin and then heat is lost by sweat evaporation. ${ }^{9}$ One of the main symptoms of POTS includes increased heart rate so the extra half-a-tablet of Metoprolol was needed to decrease the patient's heart rate when exercising in hot conditions. Metoprolol is a beta- 1 adrenergic receptor blocker which decreases one's heart rate by decreasing the force of contraction in the heart. ${ }^{10}$ This allows the blood to flow easier, preventing tachycardia. When exercising in hot conditions, sweating and tachycardia is exacerbated warranting the need for an extra half-a-tablet of metoprolol for this patient.

The patient came from a healthy, supportive family who encouraged her to do what she loves. In the patient's mind, quitting was not an option and she was willing to do what it took to compete while staying healthy. The patient was diligent in taking her medications and performing modifications if needed. The communication

Copyright $(\subset)$ by Indiana State University All rights reserved. ISSN Online 2577-8188 between physician, athlete, parents, and the athletic trainer was strong, which helped the patient to manage her conditions while still participating in her sport.

\section{INTER VENTIONS}

Upon being accurately diagnosed with POTS, the patient was prescribed Metoprolol to aid in slowing her heart rate and fludrocortisone to increase blood volume. The patient was asymptomatic at rest and during daily activities. During and after exercise is when the patient's symptoms become problematic. It was recommended that she prolong her cross-country race finish to slowly decrease her heart rate without a sudden drop in blood pressure. Due to the nature of a cross country race, the athletes have approximately $10-20$ yards to stop following crossing the finish line. This made it difficult for the athlete to slowly decrease her speed and heart rate without a sudden stop. The coach, patient and her parents, and the athletic trainer discussed the patient's situation and needs and it was determined that an assistant coach would provide support to the patient at the finish line by catching her at the conclusion of her races. After catching the patient at the finish line, the coach would lay her down and the athletic trainer would help to monitor her heart rate and oxygen levels. Once the patient's heart rate was below 130 beats per minute, she was able to stand. Following each race, the patient's heart rate returned to normal and she recovered approximately 10 minutes after finishing the race.

\section{OUTCOMES}

This patient's freshman and sophomore years of high school athletics were challenging in managing her symptoms until a correct diagnosis was obtained. When the correct doses of medication were prescribed and the patient and her healthcare team better understood her condition, the patient did not experience any episodes of 
syncope while participating in basketball. In her junior and senior year, the episodes of syncope were only experienced after completing a crosscountry race. Her coach was able to catch her following her finish. The coach and athlete would monitor her heart rate and oxygen levels via pulse oximeter. Support provided by the cross-country coaches and the athletic trainer at the finish line helped her to recover quickly and she was able continue competing throughout her high school career.

\section{DISCUSSION}

This case is unique because it is endurance and adrenaline driven. In fact, $50 \%$ of POTS patients show signs of a hyperadrenergic or high adrenaline state. ${ }^{11}$ Hyperadrenergic POTS patients have high levels of nor-epinephrine in their blood which are natural stimulants in the body. ${ }^{11}$ This can lead to an increase in heart rate and blood pressure. This patient experienced syncope episodes following long-endurance crosscountry races due to the abrupt stop at the finish line and resultant rapid drop in heart rate. Her tilt table test showed that she has hyperadrenergic POTS because her results varied after the sympathetic drug, isoproterenol, was introduced. Additionally, the patient would always pass the finish line before fainting, supporting the hyperadrenergic POTS diagnosis. Following the correct diagnosis and dosages for the patient's medication, she would only faint after running a cross-country race. During cross country practices or basketball games, the patient was able to slowly decrease activity and reduce her heart rate through breathing techniques. During basketball games and practices, she was able to tell when her heart rate was too high, so she would let her coach know she needed a substitution. After coming out of the game or practice she would sit on the bench and hydrate until her heart rate was under control.

Copyright $(\subset)$ by Indiana State University All rights reserved. ISSN Online 2577-8188
Compared to other cases, this patient had a mild case of POTS, which was managed through medications and modifications to sport participation. Some cases of POTS are more severe and have a greater effect on the patient's daily activities. For example, two cases were presented detailing the symptoms of two young, Caucasian females. The first case included a 20year-old female who showed acute onset episodes of fainting upon sitting up, dizziness, slowing of speech, and the inability to contract muscles in the bladder. ${ }^{12}$ This patient was treated with $20 \mathrm{mg}$ of propranolol, which is similar to the beta blocker the patient in the current case was prescribed. With the help of the medication, the patient only had short periods of dizziness following the diagnosis. ${ }^{12}$ The second case included a 19-year-old who showed acute onset of dizziness with syncope events. ${ }^{12}$ This patient was first treated with fludrocortisone and compression stockings. The patient also received $500 \mathrm{mg}$ of methylprednisolone for five days for suspected autonomic neuropathy. ${ }^{12}$ These two cases were unique because they started with an acute onset and the symptoms were fully disabling. Variation in onset, intensity and type of symptoms in those experiencing POTS could make diagnosis difficult. If POTS is not properly treated, the syndrome can greatly impact not just athletic activity, but activities of daily living. Proper communication between providers on the healthcare team can speed the delivery of an accurate diagnosis, aid in the development of a patient care plan, and assist the athlete in managing their symptoms.

This case presented with strengths and limitations. Overall, the patient and her family were very well informed and cooperative in providing information to inform this case. For being so young in experiencing a health condition such as POTS, this patient was a very good communicator and became an advocate for her own healthcare. Educating and empowering high school patients to communicate and manage their own health 
conditions is extremely important. Athletic trainers, parents, and coaches can work together to help the high school patient-athlete in how to best manage their condition to be able to compete at a desired level. This patient took time to understand her condition, understand her condition, learned how to manage her symptoms, and she communicated well with all athletic trainers involved in her care - at home, and while traveling for competitions.

\section{CLINICAL BOTTOM LINE}

Although POTS is not extremely prevalent in athletic training clinical practice, it is vital for athletic trainers to be educated on the signs and symptoms of POTS and understand that it can affect each patient differently. The signs and symptoms of POTS may mimic many other pathologies which may lead to misdiagnoses and ineffective treatments. Athletic trainers should urge patients and their parents to seek several opinions when symptoms include light-headedness, fatigue, sweating, anxiety, palpitations, exercise intolerance and syncope or near-syncope when standing or after exercise. When correctly diagnosed, POTS can be managed with patient education, advocacy and strong communication. Further, collaboration between healthcare providers to create an individual plan for the patient can also contribute to the management of POTS. In this case, the patient's cardiologist, parents, coaches, athletic trainers, and the patient were all closely involved in her care plan. With a few simple modifications to an athlete's procedure, close monitoring by athletic trainers, and routine checkups with a cardiologist, POTS symptoms can be managed and patientathletes can continue competing at high levels in various endurance sports.

\section{REFERENCES}

1. Agarwal AK, Garg R, Ritch A, Sarkar P. Postural orthostatic tachycardia syndrome. Postgrad Med J. 2007;83(981):478-480.

Copyright $\left({ }^{\circ}\right.$ by Indiana State University All rights reserved. ISSN Online 2577-8188 https://doi.org/10.1136/pgmi.2006.05504 6.

2. Grubb BP. Postural tachycardia syndrome. Circulation. 2008;117(21):2814-2817. https://doi.org/10.1161/circulationaha.107 .761643 .

3. Grubb BP, Kanjwal Y, Kosinski DJ. The postural tachycardia syndrome: a concise guide to diagnosis and management. J Cardiovasc Electrophysiol. 2006;17(1):108$112 . \quad$ https://doi.org/10.1111/i.15408167.2005.00318.x.

4. Benarroch EE. Postural tachycardia syndrome: a heterogeneous and multifactorial disorder. Mayo Clin Proc. 2012;87(12):1214-1225. https://doi.org/10.1016/i.mayocp.2012.08 .013.

5. Sletten DM, Weigand SD, Low PA. Relationship of $Q$-sweat to quantitative sudomotor axon reflex test (QSART) volumes. Muscle Nerve. 2010;41(2):240-246. https://doi.org/10.1002/mus.21464.

6. Illigens BM, Gibbons $\mathrm{CH}$. Sweat testing to evaluate autonomic function. Clin Auton Res. 2009;19(2):79-87. https://doi.org/10.1007/s10286-0080506-8.

7. Fedorowski A. Postural orthostatic tachycardia syndrome: clinical presentation, aetiology and management. J Intern Med. $2019 ; 285(4): 352-366$.

https://doi.org/10.1111/ioim.12852.

8. Waxenbaum JA, Reddy V, Varacallo $M$. Anatomy, Autonomic Nervous System. Treasure Island (FL): StatPearls Publishing; 2021.

https://www.ncbi.nlm.nih.gov/books/NBK53 9845/.

9. Greaney JL, Kenney WL, Alexander LM. Sympathetic regulation during thermal stress in human aging and disease. Auton Neurosci. 2016;196:81-90.

https://doi.org/10.1016/i.autneu.2015.11. $\underline{002}$. 
10. Information NCfB. PubChem Compound Summary for CID 4171, Metoprolol. https://pubchem.ncbi.nlm.nih.gov/compound Metoprolol. Accessed October 14, 2020.

11. Arnold AC, Ng J, Raj SR. Postural tachycardia syndrome - Diagnosis, physiology, and prognosis. Auton Neurosci. 2018;215:3-11. https://doi.org/10.1016/i.autneu.2018.02. $\underline{005}$.

12. Chung HY, Essig F, Wickel J, et al. Acute onset and severe manifestation of postural orthostatic tachycardia syndrome - Two cases. Clin Neurophysiol. 2020;131(1):158-159. https://doi.org/10.1016/i.clinph.2019.11.0 이. 